\title{
A Theorem on Bordering Symmetrical Determinants whose Elements are of the form $a_{a}^{r} a^{a}{ }_{s}$
}

\author{
By J. J. Nassau.
}

(Received and Read 2nd June 1928.)

1. The following is a generalization of a theorem stated by Professor H.S. Uhler and demonstrated by the writer in the American. Mathematical Monthly of October 1927.

Let $N$ denote the bordered symmetrical determinant

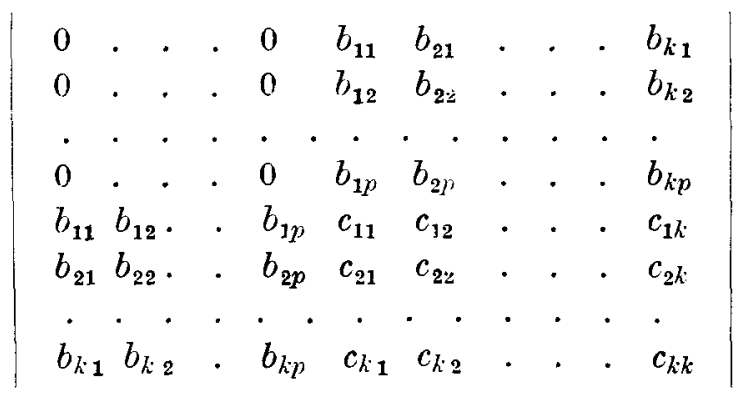

where $n>k, k>p$ and $c_{r s}=\sum_{j=1}^{j=n} a_{r, j} a_{s, j}$ and the " $a$ "'s belong to the matrix

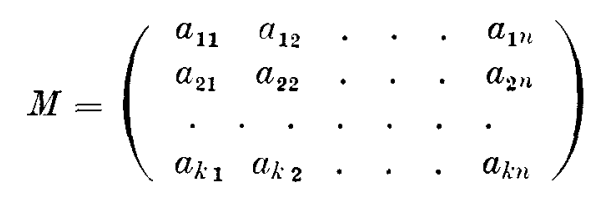

Then $N$ equals $(-1)^{p}$ times the sum of the squares of $\left(\begin{array}{c}n \\ k-p\end{array}\right)$ determinants of order $k$ every one of which has for the first $k$ columns the matrix

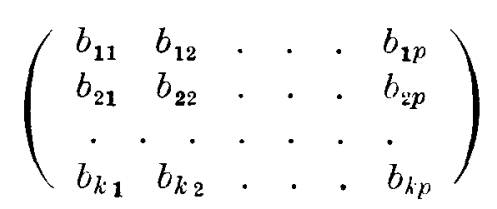

and the remaining $k-p$ monomial columns eonstitute collectively one of the combinations of $n$ columns of the matrix $M$ taken $k-p$ at a time. 
2. In as much as the generality of the proof is not destroyed, for simplicity a special value of $p$ is chosen. Let $p=2$, and denote

$$
d_{r s} \equiv \sum_{j=1}^{j=p} b_{r, j} b_{s, j} .
$$

The result of compounding the matrix

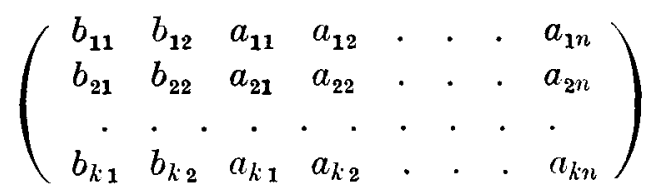

with its conjugate is a square matrix. The determinant $D_{b}{ }_{11}{ }^{b}{ }_{12}$ of this matrix $^{1}$ is

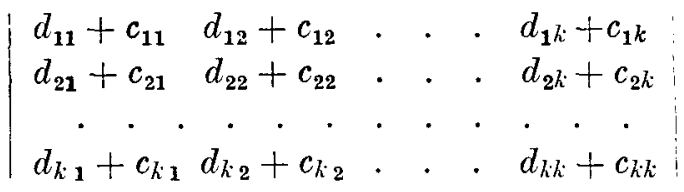

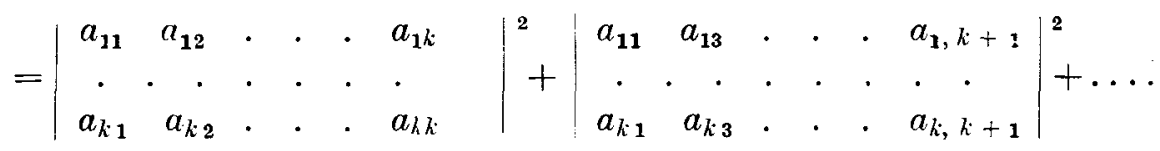

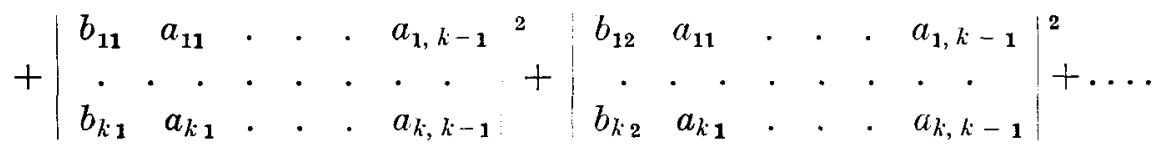

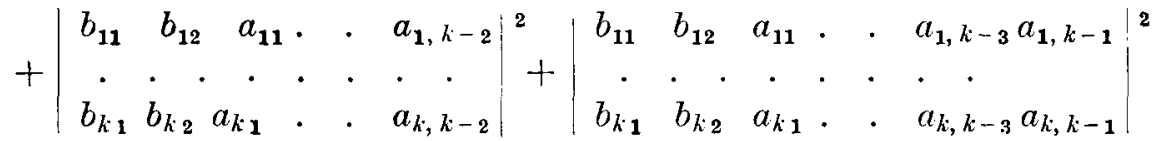

3. The left-hand side of (1) is composed:

First, of a determinant without any $d$ 's, that is $\left|\begin{array}{lll}c_{11} & c_{22} & \ldots \\ c_{k k}\end{array}\right|$.

Secondly, the sum of $k p$ determinants derived from determinants having each one column of $d$ 's. That is

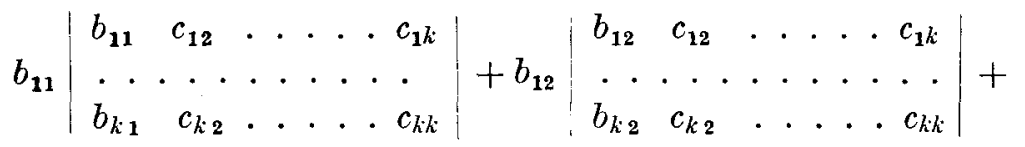

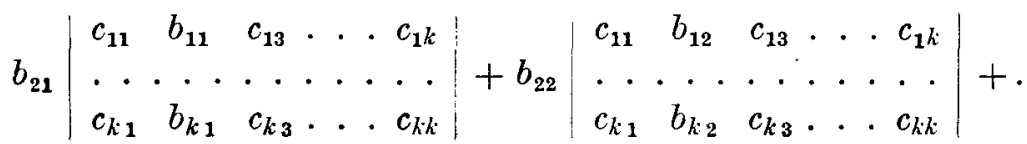

\footnotetext{
1 Scott and Mathews, Theory of Determinants, p. 54.
} 
If the 1st, $(p+1),(2 p+1) \ldots$ terms are grouped and the $2 \mathrm{nd}$, $(p+2),(2 p+2) \ldots$ terms are grouped likewise, the above sum takes the form

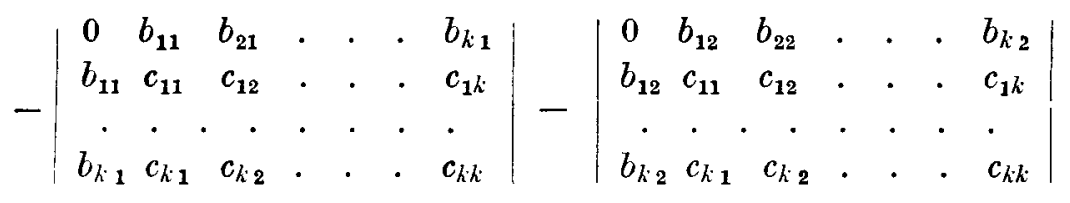

By the theorem referred to in the beginning of this paper, the last two determinants are equivalent to the sum

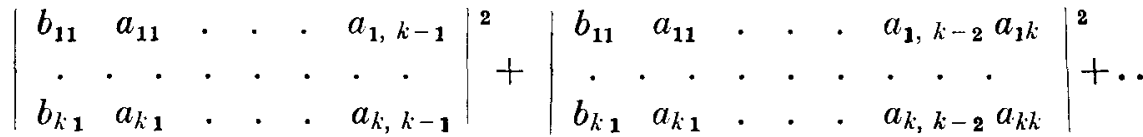

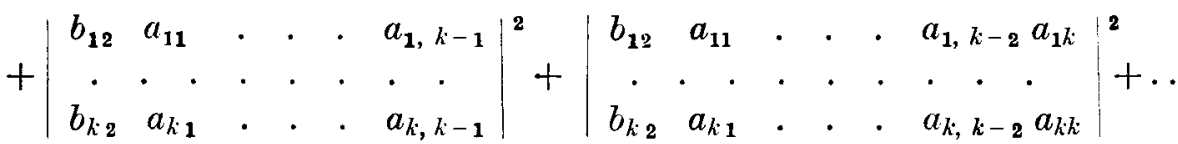

Thirdly, of the sum of $2 !\left(\begin{array}{l}k \\ 2\end{array}\right)$ determinants derived from determinants having each two columns of " $d$ "'s at a time, that is:

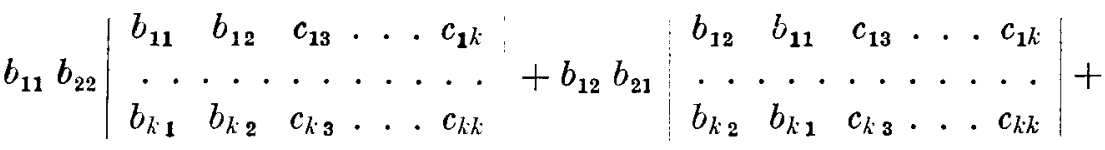

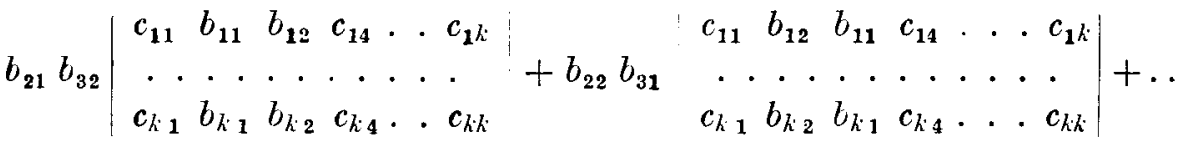

from which we obtain

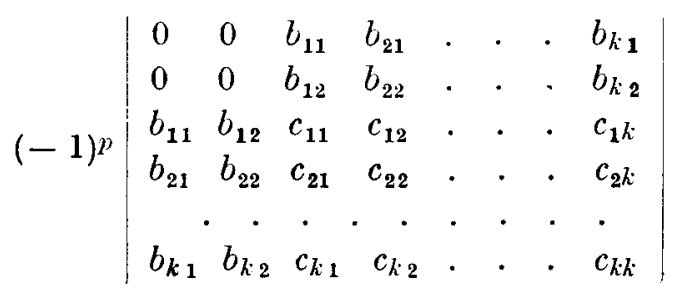

Lastly, all the determinants having $p+1$ or more, columns of $d$ 's are equal to zero, for they will have two or more columns the same. 
4. The right-hand side of (1) is composed, first of the sum of $\left(\begin{array}{l}n \\ k\end{array}\right)$ determinants without $b$ 's and is equivalent to the determinant of the square matrix obtained by compounding the matrix

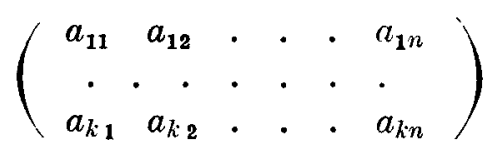

with its conjugate; and hence is equal to $\left|c_{11} c_{22} \ldots c_{k k}\right|$. This corresponds to the first determinant considered on the left side of (1).

The second group of $p\left(\begin{array}{c}n \\ k-1\end{array}\right)$ determinants of the right side, having each one column of $b$ 's, is equivalent to (2). Therefore the remaining terms of the right side are equivalent to (3), the only determinant left on the left side. Hence the theorem. 